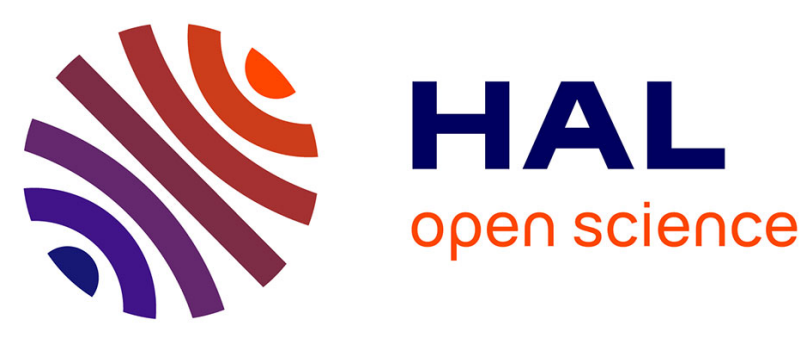

\title{
Dynamical Models for Eddy Current in Ferromagnetic Cores Introduced in an FE-Tuned Magnetic Equivalent Circuit of an Electromagnetic Relay
}

Fabien Sixdenier, Marie-Ange Raulet, Romain Marion, René Goyet, Guy Clerc, Farid Allab

\section{To cite this version:}

Fabien Sixdenier, Marie-Ange Raulet, Romain Marion, René Goyet, Guy Clerc, et al.. Dynamical Models for Eddy Current in Ferromagnetic Cores Introduced in an FE-Tuned Magnetic Equivalent Circuit of an Electromagnetic Relay. IEEE Transactions on Magnetics, 2008, 44 (6), pp.866-869. 10.1109/TMAG.2008.916431 . hal-00258255

\section{HAL Id: hal-00258255 https://hal.science/hal-00258255}

Submitted on 27 Aug 2008

HAL is a multi-disciplinary open access archive for the deposit and dissemination of scientific research documents, whether they are published or not. The documents may come from teaching and research institutions in France or abroad, or from public or private research centers.
L'archive ouverte pluridisciplinaire HAL, est destinée au dépôt et à la diffusion de documents scientifiques de niveau recherche, publiés ou non, émanant des établissements d'enseignement et de recherche français ou étrangers, des laboratoires publics ou privés. 


\title{
Dynamical Models for Eddy Current in Ferromagnetic Cores Introduced in an FE-Tuned Magnetic Equivalent Circuit of an Electromagnetic Relay
}

\author{
Fabien Sixdenier ${ }^{1}$, Marie-Ange Raulet ${ }^{1}$, Romain Marion ${ }^{1}$, René Goyet ${ }^{1}$, Guy Clerc ${ }^{1}$, and Farid Allab ${ }^{2}$ \\ ${ }^{1}$ Université Lyon 1, Villeurbanne F-69622, France \\ ${ }^{2}$ Schneider Electric 31, Eybens 38320, France
}

\begin{abstract}
Electromagnetic relay modeling is elaborated using a magnetic equivalent circuit (MEC). The lumped parameters of the MEC are fitted with respect to 3-D finite-element simulation by using classical optimization algorithms. An accurate dynamic material law has to be taken into account in the modeling, considering the massive core of the circuit. Two accurate dynamical models for representing eddy currents are studied. The simulation of the relay is carried out for several excitation frequencies. A comparison between measurements and simulated quantities is provided.
\end{abstract}

Index Terms-AC circuit breakers (CBs), eddy currents, magnetic hysteresis, optimization methods.

\section{INTRODUCTION}

$\mathbf{T}$ HE differential circuit breaker (CB) is essential to ensure life safety. Such a device is composed of three parts: the differential current sensor [1], the electronic circuit, and the electromagnetic relay. To reduce the problem, we focus on the relay that is supposed to be closed. The elaboration of a new prototype requires accurate models [2]. A 3-D field calculation, including accurate dynamical material laws [3] and a coupling with the electrical circuits, would ideally suit the problem, but would lead to prohibitive computation times. Hence, we have chosen a different approach, by considering magnetic equivalent circuits (MECs) [4] with accurate dynamical behavior of the magnetic material. The development of this model requires two successive steps:

1) elaboration of the flux tubes network;

2) introduction of dynamical effects due to eddy current in the different magnetic flux tubes.

An optimization of the lumped parameters of the flux tube network based on automatic algorithms is presented. Two accurate dynamical laws of the magnetic material are considered [5], [6] and tested. Once the association of different flux tubes is carried out, simulations of the relay are performed at several frequencies. An experimental validation will feed the discussion.

\section{DESCRIPTION OF THE RELAY}

The electromagnetic relay is composed of a magnet, a massive circuit, a mobile vane linked to a spring, and a coil. Fig. 1 shows the geometry of the relay (the scales are not respected) with its different parts.

In the initial configuration, the mobile vane is closed. The mobile vane is held in this position thanks to the magnet attraction. When a current appears in the coil, the force created by the spring becomes inferior to the one created by the magnet and the coil; thus, the relay trips.

\section{A. Modeling of the Device}

Thanks to 3-D nonlinear FE model results, an a priori MEC is defined. Fig. 2 shows the topology of the flux tubes network. Each part of the relay (magnet, magnetic circuit, air gaps, etc.)

Digital Object Identifier 10.1109/TMAG.2008.916431

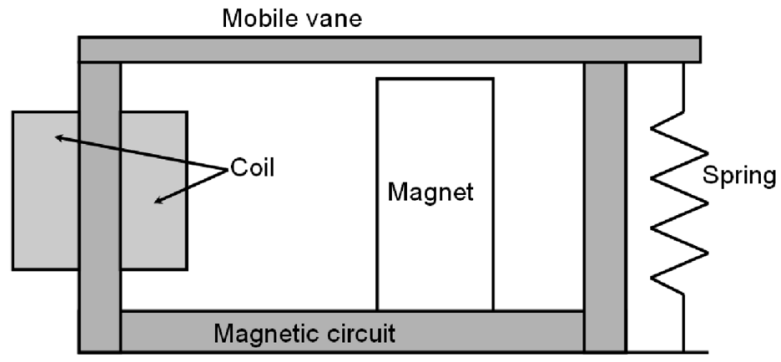

Fig. 1. Geometry of the relay.

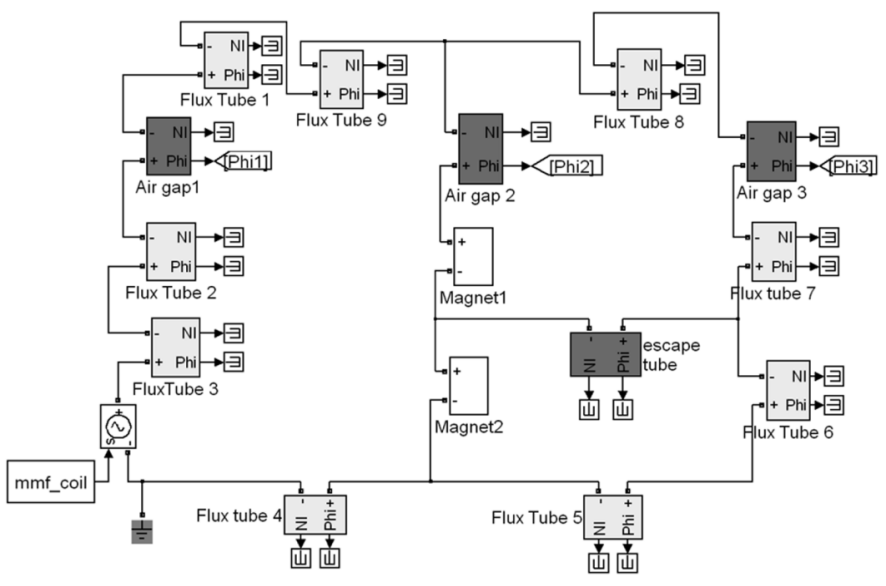

Fig. 2. MEC of the relay.

is described as a component which requires a set of data. Every flux tube is parameterized by its dimensions (length, section, depth) and its magnetic behavior (linear, nonlinear, dynamical effects or not).

This network contains three kinds of flux tubes.

1) Air-gap or leakage flux tube (three air-gaps flux tubes that are denoted air gap 1... are considered in (Fig. 2); these air gaps are located at the junctions of the mobile vane and the massive circuit).

2) Magnet flux tube: the magnet is split into two tubes (Magnet1, Magnet2) because of the leakage flux tube (escape tube on Fig. 2).

3) Magnetic flux tube (nine flux tubes must be considered to ensure good accuracy). 
Each flux tube constitutes a "magnetic circuit component" which must be linked to other magnetic or electrical circuit components [3]. This association fulfills the nodes law (1) and the Ampere's theorem (2) obtained by analogy between magnetic and electric circuits. The coupling variables are $d \phi / d t$ and $H . l$ (where $\phi$ is the magnetic flux, $H$ is the excitation field on the surface of the flux tube, $l$ is the mean length of the tube, and $N_{k} . i_{k}$ is the $k$ th MMF source)

$$
\begin{aligned}
\sum_{i=1}^{n} \frac{d \phi_{i}}{d t} & =0 \\
\sum_{j=1}^{n} H_{j} . l_{j} & =\sum_{k=1}^{n} N_{k} . i_{k} .
\end{aligned}
$$

The magnet characteristics (remanent flux density $B_{r}$ and relative permeability $\mu_{r}$ ) constitute essential data, which define the conditions of tripping the relay. If these data are known, the major criteria are the dimensions of the air gaps and the determination of the leakage flux tubes.

\section{B. Model Parameters Optimization}

In order to determine these parameters, optimization algorithms are used (Nelder-Mead's simplex, genetic algorithm). The objective function (for both algorithms) is the quadratic error between the resulting mechanical torque on the mobile vane obtained by 3-D FE and MEC models. The fitting parameters are the sections of the air gaps and of the leakage flux tubes. These parameters are initially determined with the method described in [4].

1) Simplex Algorithm: The Nelder-Mead simplex method (NMS) is a very powerful local descent direct search method for minimizing a real-valued function $f(x)$ for $x \in \Re$. In each iteration, NMS begins with a simplex, specified by its $n+1$ vertices and the associated function values. One or more test points are computed, along with their function values. At the end of each iteration, a new (different) simplex is obtained, so as to satisfy some descent conditions regarding the values of the objective function.

2) Genetic Algorithm: At first, $N$ individuals (the population) are created according to a random process. Each individual takes a set of the fitting parameters. Then, in an iterative way, the "fitness" (= objective function) is computed for all individuals, and couples of individuals are "mixed" together so as to obtain a better population, by using "genetic operators" in a way inspired by natural evolution of living organisms.

\section{Optimization Results}

Fig. 3 shows the normalized mechanical torque applied on the vane as a function of the imposed remanent flux density $B_{r}$ computed by using 3-D FE $\left(T_{\mathrm{FE}}\right)$, MEC with initial parameters $\left(T_{\text {init }}\right)$, and optimized parameters with NMS $\left(T_{\text {simplex }}\right)$ and genetic algorithm $\left(T_{\mathrm{ga}}\right)$. One sees that optimized parameters provide a much better fit with FE than initial (not optimized) parameters. In fact, the quadratic error (by taking the FE as reference) has gone up from 24 (for nonoptimized parameters) to 0.24 (simplex) and $0.43(\mathrm{GA})$, justifying the fact that the model parameters had to be optimized. The genetic algorithm was not essential; nevertheless, it ensures that the simplex

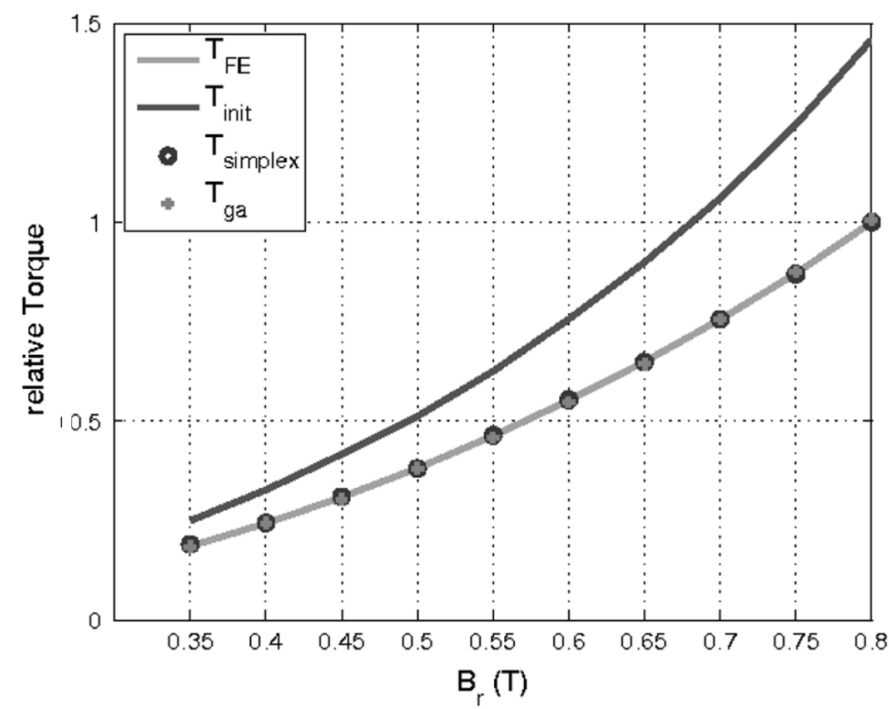

Fig. 3. Normalized torque versus magnetic flux density of the magnet.

algorithm does not fall into a local minimum. One observes that GA and NMS provide results with comparable accuracy.

\section{MAGNETIC COMPONENT}

\section{A. Elaboration of Magnetic Components}

Each box in Fig. 2 ("components") represents a magnetic flux tube. The inputs and outputs which allow the different couplings are $d \phi / d t$ and H.l. A dynamical behavioral material model must be considered, so as to define the relation between these inputs and outputs. The core which constitutes the relay is thick and the magnetic material is hardly excited (high frequency); a lot of dynamical effects induced in the core can expand. Due to the circuit thickness, Eddy currents are developed in most parts of the circuit. Therefore, an accurate material law has to be taken into account.

Two different models for representing the dynamical effects due to the eddy currents are considered. Both models have to satisfy assumptions related to each basic flux tube, and the same common assumptions can be defined: 1) The study domain is a rectangular sheet where edge effects are neglected, 2) the thickness $d$ of the sheet is much smaller than its width, 3 ) the magnetic field is supposed to be unidirectional (along the length of the sheet), and 4) the material is isotropic and its conductivity $\sigma$ is homogeneous.

The first model comes from the magnetic-field diffusion (3) reduced to a 1-D formulation [5], [7], [8]

$$
\operatorname{curl}(\operatorname{curl} H)=-\sigma \frac{\partial B}{\partial t}
$$

Due to the symmetry of the system, this equation is solved numerically by using finite element or finite difference methods on the half-thickness $(d / 2)$ of the cross section of the sheet. This model is very well suited for representing dynamical effects due to eddy currents in a sheet, provided that an accurate static material law is available. To this aim, the Jiles-Atherton static model is introduced in the diffusion problem for representing the static 
hysteresis. The resolution of this diffusion problem allows obtaining local information in the section, which is not accessible by measurement. This model named "local model" obviously considers the skin effect developed in a flux tube. In order to obtain this information, a nonlinear algebraic system has to be solved. This system contains as many equations as the number of spatial discretization nodes of the studied domain. The computation time depends on this spatial discretization and, above all, on the static magnetic model which is used. The second model arises out of the first one. A simple method to homogenize the magnetic diffusion equation along the study domain $(d / 2)$ is developed in several works [6], [7]. This homogenization allows obtaining a simple (4) associating the output and input of the searched magnetic component

$$
H_{\text {edd }}=H_{\text {stat }}\left(B_{a}\right)+\frac{\sigma \cdot d^{2}}{12} \cdot \frac{\partial B_{a}}{\partial t}
$$

where $B_{a}$ is the averaged flux density over the cross section of the sheet, $H_{\text {edd }}$ is the excitation field on the surface of the sheet, $H_{\text {stat }}\left(B_{a}\right)$ is the magnetization field associated with $B_{a}$ calculated from the static material law (the relation between $B_{a}$ and $H_{\text {stat }}$ is provided by Jiles-Atherton's static hysteretic model). This model, named "global," allows computing the averaged flux density as a function of the surface excitation field. The skin effect is obviously neglected. The relevance of this model is to require few computation times, conversely to the "local" model. Minor improvements of both models allow obtaining two different dynamical magnetic components.

\section{B. Experimental Validation}

Two dynamical magnetic components are available. In order to choose the more suitable one to modelize the problem, tests of both models have been performed on a sample made of the same material as the relay core. The sample is made of a stack of rings. The thickness of each ring is the same as the thickness of the core building the relay in the aim to respect the development of dynamical effects. The material of the ring is made of $\mathrm{NiFe}$, its conductivity is $2080.10^{3} \mathrm{~S} / \mathrm{m}$, and the thickness is higher than $1 \mathrm{~mm}$. Tests are performed by considering a $150-\mathrm{Hz}$ frequency sinusoidal magnetization field applied to the surface of the sample. Fig. 4 compares the measured dynamical loops with the ones computed by the "global" and "local" models.

The "local" magnetic component obviously represents the dynamical behavior of the sample more accurately. The skin depth can be estimated to be $0.2 \mathrm{~mm}$. In this case, the magnetic field is not homogeneous in the cross section of the sample. The validity domain of the global model is overshot. Fig. 5 confirms this result by comparing the temporal values of the magnetization fields applied to the surface of the sample, and calculated in the middle of the section $(d / 2)$ with the local model.

\section{RELAy SimUlation AND EXPERIMENTAL VALIDATION}

\section{A. Experimental Protocol}

In the aim to validate the relay model, experiments have been performed on the device for different frequencies sine current excitation. For each test performed, the relay is fed with a sine source voltage of amplitude $10 \mathrm{~V}$ serially a $1-\mathrm{k} \Omega$ resistor. The

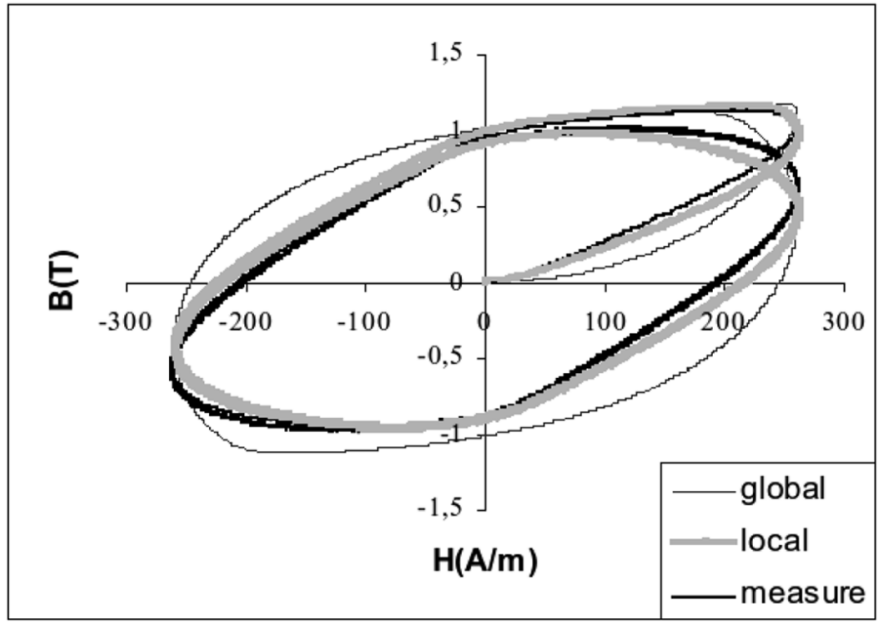

Fig. 4. Dynamical loops: measured and calculated (with "global" and "local" models), 150-Hz frequency operation.

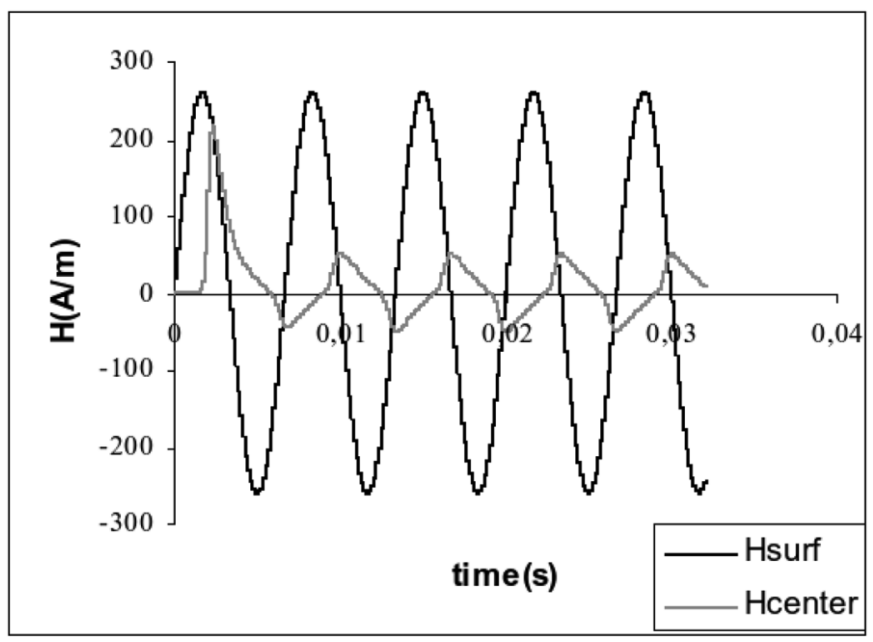

Fig. 5. Temporal evolutions of the magnetization fields on the surface and in the middle of the depth for $150-\mathrm{Hz}$ frequency operation.

waveforms of the current and of the induced electromotive force in the coils are measured.

\section{B. Temporal Waveforms}

Figs. 6 and 7 show the comparison between the measured and simulated data by considering both dynamical components for $50-\mathrm{Hz}$ and $400-\mathrm{Hz}$ excitation frequencies. $V_{m}$ and $I_{m}$ are, respectively, the measured voltage and current. $V_{s-\text { global }}$ and $V_{s-\text { local }}$ are, respectively, the voltages calculated with both global and local models, considering the same current as the measured one.

For a low frequency $(50 \mathrm{~Hz})$, both models suit the problem; the global model gives accurate results because the skin effect is not too important. However, for a high frequency $(400 \mathrm{~Hz})$, the local model improves the accuracy of the results: the validity domain of the global model is reached.

\section{Frequency Behavior}

An essential information for relay designers is the estimation of the tripping current as a function of the frequency excita- 


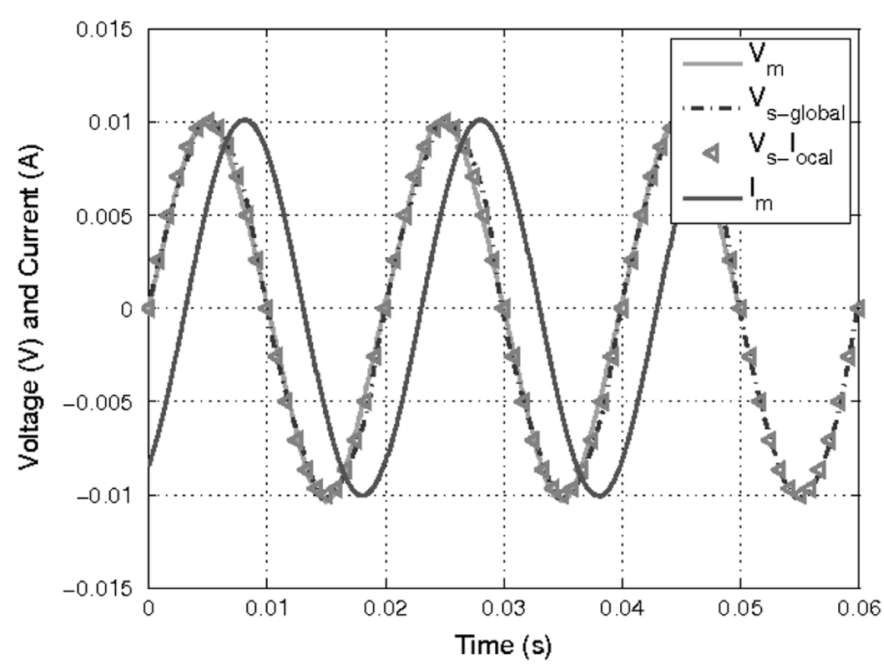

Fig. 6. Measured and simulated voltages of the coil for a sine current, $50-\mathrm{Hz}$ frequency operation.

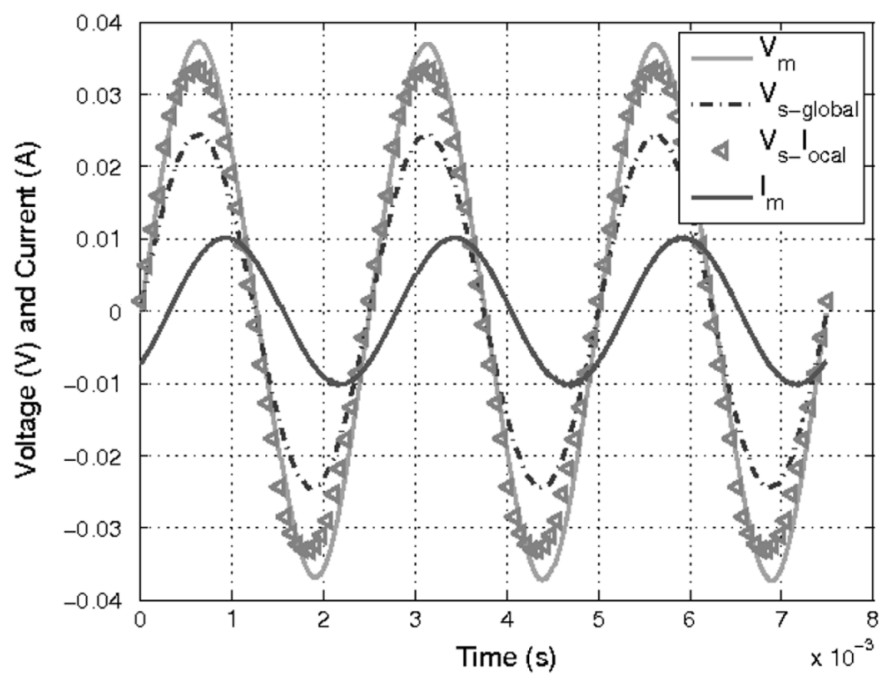

Fig. 7. Measured and simulated voltages of the coil for a sine current, $400-\mathrm{Hz}$ frequency operation.

tion. The nominal tripping current $\left(I_{n}\right)$ is defined for $50-\mathrm{Hz}$ frequency operation. Fig. 8 compares the measured and calculated factor $I_{\text {trip }} / I_{n}$ with both dynamical models, for several excitation frequencies. One observes that as in the previous case, the global model provides good results for low frequencies, but fails at higher frequencies because the skin effect is no longer negligible.

\section{CONCLUSION}

A dynamical model of an electromagnetic relay is elaborated and analyzed in this paper. For practical applications, this relay has to be associated with a differential CB. In the aim to allow the coupling between the relay model and the electrical circuit equations, an approach of the problem by using an MEC is chosen. The dynamical material behavior must be taken into ac-

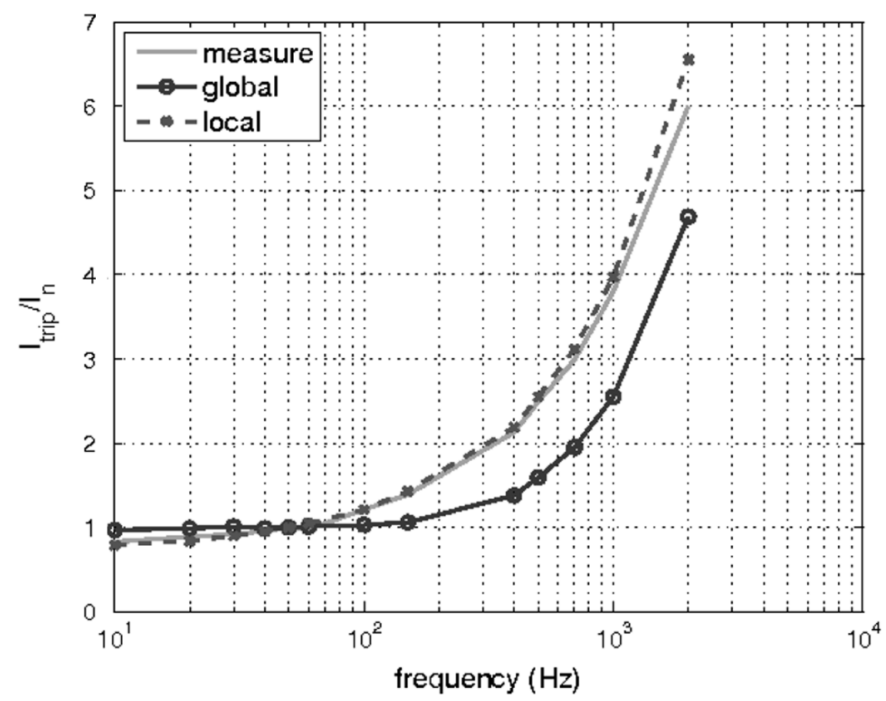

Fig. 8. Tripping factor $I_{\text {trip }} / I_{n}$ versus the frequency.

count accurately, in view of the massive cores building the relay. Two different dynamic magnetic components, both able to represent the dynamic behavior of a flux tube, are presented and tested on a sample. Simulations of the relay are carried out for different working frequencies. The results are quite correct for design purposes, and the computation time remains moderate. Works about the application of the proposed model to represent the dynamical behavior of an industrial differential $\mathrm{CB}$ are in progress.

\section{REFERENCES}

[1] B. Colin, C. Chillet, A. Lebouc, and P. Mas, "Effects of magnetic core geometry on false detection in residual current sensor," J. Magnet. Magn. Mater., vol. 304, no. 2, pp. e804-e806, Sep. 2006.

[2] J. D. Williams, R. Yang, and W. Wang, "Numerical simulation and test of a UV-LIGA-fabricated electromagnetic micro-relay for power applications," Sensors Actuators A: Phys., vol. 120, no. 1, pp. 154-162, Apr. 29, 2005.

[3] V. Silva, G. Meunier, and A. Foggia, "A 3D finite element computation of eddy currents and losses in the stator and laminations of large synchronous machines," IEEE Trans. Magn., vol. 32, no. 3, pp. 1569-1572, May 1996.

[4] V. Ostovic, Dynamics of Saturated Electric Machines. Berlin, Germany: Springer-Verlag, 1989.

[5] R. M. Del Vecchio, "An efficient procedure for modeling complex hysteresis processes in ferromagnetic materials," IEEE Trans. Magn., vol. MAG-16, no. 5, pp. 809-811, Sep. 1980.

[6] A. J. Bergqvist and S. G. Engdahl, "A homogeneization procedure of field quantities in laminated electrical steel," IEEE Trans. Magn., vol. 37, no. 5, pp. 3329-3331, Sep. 2001

[7] J. J. C. Gyselink, L. Vandevelde, D. Makaveev, and J. A. Melkebeek, "Calculation of non-load losses in an induction motor using an inverse vector Preisach model and eddy-current loss model," IEEE Trans. Magn., vol. 36, no. 4, pp. 856-860, Jul. 2000.

[8] M. A. Raulet, B. Ducharne, J. P. Masson, and G. Bayada, "The magnetic field diffusion equation including dynamic hysteresis: A linear formulation of the problem," IEEE Trans. Magn., vol. 40, no. 2, pp. 872-875, Mar. 2004.

Manuscript received June 24, 2007. Corresponding author: F. Sixdenier (e-mail: fabien.sixdenier@univ-lyon1.fr). 